\title{
STAR FORMATION AND THE ORIGIN OF STELLAR MASSES
}

\author{
PH. PODSLADLOWSKI AND N. M. PRICE \\ Institute of Astronomy, Madingley Road, Cambridge, England
}

\begin{abstract}
We present a new model to explain stellar mass distributions in different stellar environments. In our model, the protostar phase is terminated, when the protostellar core embedded in a molecular clump experiences a collision with another star or protostellar clump, which ejects the protostellar core from its parent clump. Such dynamical interactions are necessarily important, if stars preferentially form in dense clusters. We show that, in a simple model, the initial mass function approaches a simple, asymptotic form, which strongly resembles observed mass functions. The model has important consequences for star formation in different environments. We also discuss the implications of the model for our understanding of pre-main-sequence stellar evolution.
\end{abstract}

\section{COLLISIONS AND THE INITIAL MASS FUNCTION (IMF)}

Modern observations of star-forming regions show that stars preferentially form in very dense, but ultimately unbound clusters (for references see Podsiadlowski and Price 1992). This suggests that collisions between young stellar objects (YSOs) are important. We have studied two types of collisions: collisions between two protostellar cores (i.e., protostars embedded in dense envelopes from which they accrete) and collisions between a protostellar core and a star (i.e., a YSO which is no longer embedded in a massive envelope). The cross section for collisions between protostellar cores is mainly determined by the geometric size of their envelopes. The most likely outcome, if the collision is supersonic, is that the protostellar cores are separated from their envelopes, thus terminating the protostar accretion phase. A collision between a protostellar core and a star will also separate a protostar from its envelope, if the kick velocity imparted to the protostar (relative to its envelope) by a star passing through the core exceeds the central escape velocity. We estimate that, for characteristic core properties, the cross section for the latter collisions is of order $10 \%$ of the cross section for direct core-core collisions. In the simulations we present below, direct core-core collisions dominate initially, until the number of stars significantly exceeds the number of protostellar cores.

To illustrate how such collisions lead to a mass spectrum, we adopt a very simple model for the star-forming process. We assume that stars form at a constant rate (per unit gas mass), $\alpha$, and that protostars accrete mass at a constant rate, $\dot{M}$, until they are ejected (the individual rates for core-core collisions and core-star collisions are $\beta$ and $\beta Z[Z \sim 0.1]$, respectively). In Figure 1 we 


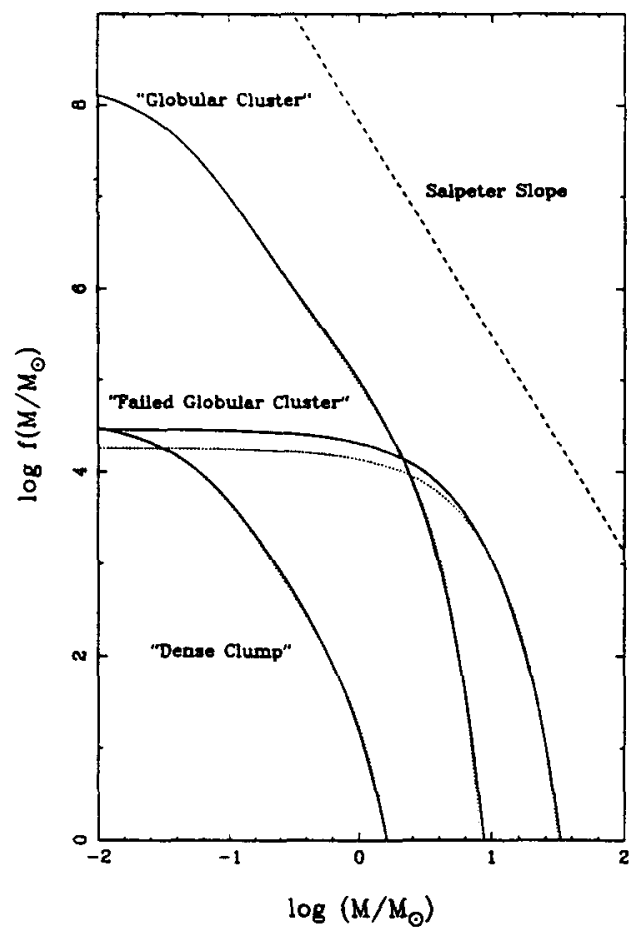

Figure 1 - IMFs resulting from a collisional model for three starformation environments, a globular cluster (GC), a "failed" GC and a dense molecular clump ("Clump"), respectively (solid curves as indicated). The "GC" and the "Clump" IMFs both have power-law segments, resembling a Salpeter IMF (shown as a dashed curve). The initial gas masses $M_{\mathrm{g}}$, in the three cases were $10^{6}, 10^{6}$ and $5 \times 10^{2} M_{\odot}$, the star-formation rates, $\alpha$, were $10^{-7}, 10^{-8}$ and $10^{-8} / M_{\odot} / \mathrm{yr}$, the collisions rates, $\beta$, were $10^{-9}, 5 \times$ $10^{-10}$ and $2 \times 10^{-7} / \mathrm{yr}(Z=0.1$ in all cases), the protostar accretion rates, $\dot{M}$, were $10^{-5}, 10^{-5}$ and $10^{-6} M_{\odot} /$ yr. At the end of the simulation (after $10^{8}, 10^{7}$ and $5 \times 10^{7}$ yrs, respectively), the gas mass fractions were $\sim 50, \sim 70$ and $\sim 70 \%$, respectively.

present the IMFs that result from this simple model for different star-forming environments, where we used parameters which may be representative for a dense molecular clump, a bound (surviving) globular cluster and a "failed" globular cluster, respectively.

One of the major results of our calculations is that the IMF asymptotically approaches a simple and universal distribution provided that star formation is relatively inefficient (which here means that the timescale on which gas is locked up in stars is longer than the core-star collision time). This asymptotic solution is of the form:

$$
f(m) d m \propto \frac{1-(1+m) e^{-m}}{m^{2}} g d m
$$

where $m \equiv M / M_{\mathrm{sc}}$ is a dimensionless mass and $g \equiv \exp \left[-\left(M / M_{\mathrm{co}}\right)^{2}\right]$ is the exponential cut-off factor ( $M$ is the mass of the star, and $M_{\text {co }}$ and $M_{\text {sc }}$ are the cut-off mass and the scaling mass respectively). For $M_{\mathrm{sc}}<M<M_{\mathrm{co}}$, the distribution approaches a Salpeter-like power law (Salpeter 1955), $f(M) d m \propto m^{-2.35} d m$, and the whole. IMF exhibits all the gross features of Scalo's mass function (Scalo 1986). While the cut-off mass depends only on intrinsic parameters ( $M_{\text {co }} \sim \dot{M} / \sqrt{\beta Z \alpha M_{\mathrm{g}}}$, where $M_{\mathrm{g}}$ is the total mass of the cloud or clump), the scaling mass decreases with time, $t,\left(M_{\mathrm{sc}} \simeq \dot{M} /\left[\beta Z \alpha M_{\mathrm{g}} t\right]\right)$. Thus the distribution more closely approaches a simple power law to lower masses the longer star formation proceeds. Although this simple model is not fully realistic, the very existence of a universal asymptotic solution may be sufficient to guarantee that the overall distribution, averaged over various populations, may still reflect the basic characteristics of the solution. 


\section{APPLICATIONS}

Our model has important implications for star formation in different stellar environments. For example, the cut-off of the distribution at high masses depends on the density of the star-forming region. In high-collision environments like globular clusters, massive stars may be unable to form (see Fig. 1). This may avoid the problem of self-pollution with heavy elements produced in the first supernovae without requiring very fast and efficient star formation in globular clusters. On the other hand, if failed globular clusters are the environment in which halo stars and population III stars are formed, their IMF should be relatively flat, i.e., biased towards high masses (see Fig. 1).

The model may also be relevant to the formation of binary and multiple systems, since core-star collisions in which the protostar is dislodged from the center of its core, but remains bound to it should be much more common than collisions which eject the protostar. After a protostar has been displaced from the center of a core, a new protostar can form in the center, which may ultimately lead to the formation of a binary.

\section{PRE-MAIN-SEQUENCE EVOLUTION}

Our model can be tested most directly - at least in principle - by comparing its predictions with the mass distributions observed in individual star-forming clusters. This requires knowledge of the mass - luminosity relation of accreting protostars to construct the protostar luminosity function (PLF; alternatively one can use this method to test protostar theory). Protostar theory is rather uncertain, since the structure of protostars depends on the accretion rate, $\dot{M}$, and is sensitive to the surface boundary condition. We have constructed a simple protostar luminosity function based on the following assumptions: (1) Protostars lie on Hayashi tracks. (2) Before deuterium burning, the mass - radius relation is determined by the condition that the accretion timescale is of the order of the Kelvin-Helmholtz time. (3) During deuterium burning, the mass - radius relation is approximately given by $R \simeq 5 R_{\odot}\left(M / M_{\odot}\right)$. With these assumptions, we can derive a theoretical birthline in the Hertzsprung-Russell diagram and a PLF. While our theoretical birthline is in good agreement with the observed birthline (also see Stahler 1988), our PLF differs substantially from the one derived by Stahler and Fletcher (1992, preprint); most importantly, it has no peak at low masses $\left(M \sim 0.1 M_{\odot}\right)$. This difference can be entirely attributed to different initial conditions for the accretion process. We emphasize that this discrepancy has to be resolved, before a meaningful comparison between our star-formation scenario and observations of star-forming clusters can be made.

\section{REFERENCES}

Podsiadlowski, Ph. and Price, N. M. 1992, Nature, in press.

Salpeter, E. E. 1955, ApJ, 121, 161.

Scalo, J. M. 1986, Fund. Cosmic Phys., 11, 1.

Stahler, S. 1988, ApJ, 332, 804. 